Ann. Scient. Éc. Norm. Sup.,

4e série, t. 40, 2007, p. 113 à 133.

\title{
FORMAL LOOPS II: A LOCAL RIEMANN-ROCH THEOREM FOR DETERMINANTAL GERBES
}

\author{
BY MIKHAIL KAPRANOV ${ }^{1}$ AND ÉRIC VASSEROT
}

AbStract. - Given a scheme $S$ and a locally free sheaf of $\mathcal{O}_{S}((t))$-modules, we construct a determinantal $\mathcal{O}_{S}^{\times}$-gerbe over $S$ whose class is computed via a local Riemann-Roch theorem of a new kind.

(c) 2007 Elsevier Masson SAS

RÉSUMÉ. - À un schéma $S$ et un faisceau localement libre de $\mathcal{O}_{S}((t))$-modules, on associe une gerbe déterminantale sur $S$ de lien $\mathcal{O}_{S}^{\times}$. La classe de cette gerbe se calcule par le biais d'un théorème "à la Riemann-Roch» d'un nouveau type.

(c) 2007 Elsevier Masson SAS

0.1. The goal of this paper and the next one [21] is to relate three subjects of recent interest:

(A) The theory of sheaves of chiral differential operators (CDO), see [16,17]. A sheaf of CDO on a complex manifold $X$ is a sheaf of graded vertex algebras with certain conditions on the graded components. As shown in loc. cit., locally on $X$ such an object always exists and is unique up to an isomorphism but the isomorphism not being canonical, the global situation is similar to the behavior of spinor bundles on a Riemannian manifold. This is expressed by saying that sheaves of $\mathrm{CDO}$ form a gerbe $\mathcal{C D O} \mathcal{O}_{X}$. A global object exists if and only if the characteristic class

$$
\operatorname{ch}_{2}(X)=\frac{1}{2} c_{1}^{2}(X)-c_{2}(X)
$$

vanishes.

(B) The theory of the group $G L(\infty)$ developed by Sato and others [27], in particular, of the determinantal central extension of $G L(\infty)$ and of the semi-infinite Grassmann manifold (the Sato Grassmannian). In fact, what appears here is a nonlinear version of this theory.

(C) The refinement of the Grothendieck-Riemann-Roch theorem found by Deligne [9]. Recall that if $\pi: \mathcal{X} \rightarrow B$ is a smooth projective morphism of algebraic varieties and $\mathcal{E}$ is an algebraic vector bundle on $\mathcal{X}$, then Grothendieck-Riemann-Roch describes the Chern classes $c_{i}\left(R \pi_{*}(\mathcal{E})\right)$ in terms of the cohomological direct images of the Chern classes of $\mathcal{E}$ and of the relative tangent bundle $\Theta_{\mathcal{X} / B}$. When $i=1$, the first Chern class in question can be seen as the class of the determinantal line bundle $\operatorname{det} R \pi_{*}(\mathcal{E})$ in the Picard group of $B$ and Deligne posed the problem of identifying this bundle itself up to a canonical isomorphism. He solved this problem for the case when $\pi: \mathcal{X} \rightarrow B$ is a family of curves.

\footnotetext{
${ }^{1}$ The research of M.K. was partially supported by an NSF grant. 
Our theory uncovers a new, deeper level of this subject: that of a "local" version of Deligne-Riemann-Roch describing determinantal gerbes, not determinantal line bundles.

0.2. The relation $(\mathrm{A})-(\mathrm{B})$ proceeds via the ind-scheme $\mathcal{L}(X)$ of formal loops introduced in [20]. It contains the scheme $\mathcal{L}^{0}(X)$ of formal arcs, see [15]. They are algebro-geometric analogs of the Frechet manifolds

$$
L X=C^{\infty}\left(S^{1}, X\right), \quad L^{0} X=\operatorname{Hol}\left(D^{2}, X\right),
$$

where $D^{2}$ is the unit disk $\{|z| \leqslant 1\}$ and Hol stands for the space of holomorphic maps smooth on the boundary.

Sato's theory of $G L(\infty)$ can be developed in two versions. The formal version works with a locally linearly compact topological vector space $V$ such as the space $\mathbb{C}((t))$ with the $t$-adic topology. The group $G L(\infty)$ is then interpreted as the group of continuous automorphisms of $V$. This is the version we adopt in the main body of the paper. The Hilbert version starts with a Hilbert space $H$ equipped with a polarization: a class of splittings $H=H_{+} \oplus H_{-}$defined up to some equivalence, see [27]. The group $G L(\infty)$ is interpreted as the group of bounded linear automorphisms preserving the polarization. In both cases one has a Grassmann-type variety $\mathcal{G}$ (the Sato Grassmannian) and a determinantal line bundle $\Delta$ on $\mathcal{G} \times \mathcal{G}$ making $\mathcal{G}$ into a set of objects of a $\mathbb{C}^{\times}$-gerbe acted upon by $G L(\infty)$.

A nonlinear version of the theory should involve infinite-dimensional manifolds with a $G L(\infty)$-structure in the tangent bundle. In the sequel to this paper [21] we develop a convenient formalization of this idea in the algebro-geometric setting. The corresponding objects are locally compact smooth ind-schemes, introduced in [20]. Their tangent spaces possess a $G L(\infty)$ structure in the formal version above. In particular, for such an ind-scheme $Y$ we have the relative Sato Grassmannian $\mathcal{G} \rightarrow Y$ and a determinantal line bundle $\Delta$ on $\mathcal{G} \times_{Y} \mathcal{G}$ which gives rise to an $\mathcal{O}_{Y}^{\times}$-gerbe $\mathcal{D} t_{Y}$. The "determinantal anomaly" of $Y$ is the class of $\mathcal{D} t_{Y}$ in the group $H^{2}\left(Y, \mathcal{O}_{Y}^{\times}\right)$classifying $\mathcal{O}_{Y}^{\times}$-gerbes. An example is provided by $Y=\mathcal{L} X$. It turns out that it is precisely this gerbe that governs sheaves of CDO. In other words, the anomaly in constructing CDO is precisely the determinantal anomaly for the loop space [21].

Our main result, Theorem 5.3.1, has the following consequence. The class $\left[\mathcal{D}_{\text {et }} \mathcal{L} X\right]_{\in} \in$ $H^{2}\left(\mathcal{L} X, \mathcal{O}^{\times}\right)$is equal to the image of the characteristic class $(0.1 .1)$ under the transgression map (tame symbol)

$$
\tau: H^{2}\left(X, K_{2}\left(\mathcal{O}_{X}\right)\right) \rightarrow H^{2}\left(\mathcal{L} X, \mathcal{O}^{\times}\right)
$$

0.3. The above identification of $\left[\mathcal{D e t}_{\mathcal{L} X}\right]$ can be seen as a particular case of a RiemannRoch-type result for determinantal gerbes, and it is this Riemann-Roch theorem for gerbes that constitutes the main result of this paper. It is easier to explain the situation in the $C^{\infty}$-framework (0.2.1). Let $\Sigma$ be an oriented $C^{\infty}$-manifold diffeomorphic to $S^{1}$ and $E$ a $C^{\infty}$ complex vector bundle on $\Sigma$. We have then the space of smooth sections $\Gamma^{C^{\infty}}(\Sigma, E)$ and its Hilbert space completion $H=\Gamma^{L^{2}}(\Sigma, E)$. The latter can be defined using any Riemannian metric on $\Sigma$ and Hermitian metric on $E$ and is independent, as a topological vector space, of the choices. It has a canonical $G L(\infty)$-structure. To define it, one realizes $\Sigma$ as the boundary of a holomorphic disk $D$ and extends $E$ to a holomorphic bundle $\mathcal{E}$ on $D$. Then one takes $H_{+}=\Gamma^{\text {hol }}(D, \mathcal{E}$ ) (holomorphic sections) and the resulting $G L(\infty)$-structure is independent on the choice of $D, \mathcal{E}$. Now, let $p: \Sigma \rightarrow B$ be a smooth $S^{1}$-fibration over a $C^{\infty}$ (or Frechet) manifold $B$ and suppose that fibers of $p$ are oriented. If $E$ is a smooth vector bundle on $\Sigma$, we have a "bundle of vector spaces with $G L(\infty)$-structure" on $B$, namely the $L^{2}$-direct image $p_{*}^{L^{2}}(E)$. It gives a gerbe $\mathcal{D}$ et $\left(p_{*} E\right)$ with

$4^{e}$ SÉRIE - TOME $40-2007-\mathrm{N}^{\circ} 1$ 
band (or "lien") $\mathcal{O}_{B}^{\times}$, the sheaf of invertible $C^{\infty}$-functions and hence gives a class

$$
\left[\operatorname{Det}\left(p_{*} E\right)\right] \in H^{2}\left(B, \mathcal{O}_{B}^{\times}\right) .
$$

Denoting by $\delta: H^{2}\left(B, \mathcal{O}_{B}^{\times}\right) \rightarrow H^{3}(B, \mathbb{Z})$ the coboundary map of the exponential sequence, we get a class $\delta\left[\operatorname{Det}\left(p_{*} E\right)\right] \in H^{3}(B, \mathbb{Z})$. For $B=L X$ the tangent bundle $\Theta_{L X}$ can be represented as $p_{*} \mathrm{ev}^{*}\left(\Theta_{X}\right)$ where in the diagram

$$
L X \stackrel{p}{\leftarrow} S^{1} \times L X \stackrel{\text { ev }}{\leftarrow} X
$$

$p$ is the projection and ev is the evaluation map. The class $\left[\mathcal{D e t}_{L X}\right] \in H^{2}\left(L X, \mathcal{O}^{\times}\right)$is a particular case of (0.3.1).

Now, the "local Riemann-Roch" for a smooth circle fibration $p: \Sigma \rightarrow B$ says that after inverting 2 , one has

$$
\delta\left[\operatorname{Det}\left(p_{*} E\right)\right]=\int_{\Sigma / B}\left(\frac{1}{2} c_{1}^{2}(E)-c_{2}(E)\right) \in H^{3}(B, \mathbb{Z}) \otimes \mathbb{Z}\left[\frac{1}{2}\right],
$$

where $\int_{\Sigma / B}: H^{4}(\Sigma, \mathbb{Z}) \rightarrow H^{3}(B, \mathbb{Z})$ is the cohomological push-forward. This theorem and its higher-dimensional version (see below) will be proved in a subsequent paper. The main result of the present paper (Theorem 5.3.1) is a formal version of (0.3.3) (with formal Laurent series replacing functions on $S^{1}$ ) for the trivial fibration.

0.4. Theorem 5.3.1 can in fact be seen as a statement comparing two central extensions of the loop group $G L_{N}((t))$ (or $L\left(G L_{N}\right)$ in the analytic setting). If $G$ is a simple, simply connected algebraic group over $\mathbb{C}$, then the group of central extensions of $G((t))$ by $\mathbb{C}^{\times}$is generated by the class of the so-called "primitive" extension which goes back to the works of Moore [25] and Matsumoto [24]. For $G=S L_{N}$ the primitive extension (or the universal central extension with center $K_{2}$ ) was related with the second Chern class by S. Bloch [3]. It is also well known in the theory of Kac-Moody groups [27,22] that the primitive extension for $S L_{N}((t))$ is precisely the determinantal one. Combined together, these observations can be used to prove a particular case of (0.3.3): that for $E$ with $\operatorname{det}(E)$ trivial, we have

$$
\delta\left[\operatorname{Det}\left(p_{*}(E)\right]=-\int_{\Sigma / B} c_{2}(E) .\right.
$$

The case of more general reductive groups $G$ is more complicated and was studied in [7] and other papers. Our result identifies the determinantal central extension of $G L_{N}((t))$ with the extension coming from $c h_{2}$. It does not directly follow from the cited papers since we need an identification as a group ind-scheme, not as a discrete group.

The relation of (0.3.3) to Deligne-RR can be understood as follows. Suppose that $B$ is a complex algebraic variety and $\Sigma$ is embedded into a family $\pi: \mathcal{X} \rightarrow B$ of algebraic curves, as in $(0.1)(C)$ so that $\mathcal{X}$ is split into the "inside" and "outside" parts: $\mathcal{X}=\mathcal{X}_{+} \cup \mathcal{X}_{-}$. Then every extension $\mathcal{E}_{+}$of $E$ to a holomorphic bundle on $\mathcal{X}_{+}$gives an object $\left[\mathcal{E}_{+}\right]$of $\operatorname{Det}\left(p_{*} E\right)$. Similarly, an extension $\mathcal{E}_{-}$of $E$ to a holomorphic bundle in $\mathcal{X}_{-}$gives another object $\left[\mathcal{E}_{-}\right]$. A choice of both $\mathcal{E}_{+}$and $\mathcal{E}_{-}$gives a holomorphic bundle $\mathcal{E}$ on $\mathcal{X}$ and

$$
\operatorname{Hom}_{\mathcal{D} e t\left(p_{*} E\right)}\left(\left[\mathcal{E}_{+}^{\prime}\right],\left[\mathcal{E}_{+}^{\prime \prime}\right]\right)=\operatorname{det}\left(R \pi_{*} \mathcal{E}\right) .
$$


So we recognize in (0.3.3) the beginning of the ch.Td quantity of the Riemann-Roch theorem.

Put differently, the relation of the Riemann-Roch theorem to (0.3.3) is similar to the relation of the self-duality of the Jacobian of a curve to the Cartier self-duality of the ind-group scheme $G L_{1}((t))$ established by C. Contou-Carrère [8]. In fact, the Contou-Carrère symbol plays an important role in our approach.

0.5. The paper is organized as follows. The first section provides a necessary reminder on gerbes and their associated cohomology classes. Section 2 gives a background on ind-schemes. In particular, we discuss what is a sheaf or a gerbe on an ind-scheme. The determinantal gerbe is defined in Section 3. In Section 4 we study some particular ind-schemes, the ind-schemes of loops, and construct the transgression map (the Contou-Carrère symbol) from K-theory of a scheme to K-theory of its loop ind-scheme. Finally, in Section 5 we introduce the version of Chern classes convenient for our purposes and prove the local Riemann-Roch theorem. The proof uses some computations of Anderson and Pablos Romo [2] together with the reduction of central extensions of $G L_{N}$ to those of $S L_{N}$ along the lines of Brylinski and Deligne [7]. The final Section 6 explains the relation of our results to the theory of chiral differential operators.

\section{Reminder on gerbes}

Recall that a ringed space is a pair $(S, \mathcal{A})$ where $S$ is a topological space and $\mathcal{A}$ is a sheaf of rings on $S$. In this paper we will assume $\mathcal{A}$ commutative. A morphism of ringed sites

$$
f:(\mathcal{S}, \mathcal{A}) \rightarrow(\mathcal{T}, \mathcal{B})
$$

is a pair consisting of a continuous map

$$
f_{\sharp}: \mathcal{S} \rightarrow \mathcal{T}
$$

and a morphism of sheaves of rings on $\mathcal{S}$

$$
f^{b}: f_{\sharp}^{-1}(\mathcal{B}) \rightarrow \mathcal{A} .
$$

If $\mathcal{M}$ is a sheaf of $\mathcal{B}$-modules on $\mathcal{T}$, we denote by

$$
f^{*} \mathcal{M}=f_{\sharp}^{-1} \mathcal{M} \otimes_{f_{\sharp}^{-1} \mathcal{B}} \mathcal{A}
$$

its inverse image in the sense of morphisms of ringed spaces. Note that we have a morphism $f^{*}: H^{i}(\mathcal{T}, \mathcal{M}) \rightarrow H^{i}\left(\mathcal{S}, f^{*} \mathcal{M}\right)$.

If $\mathcal{S}$ is a topological space and $\mathcal{F}$ is a sheaf of abelian groups on $\mathcal{S}$, then we can speak of $\mathcal{F}$-gerbes (= gerbes with band or "lien" $\mathcal{F}$, see [6]). Recall that such a gerbe $\mathcal{G}$ consists of the following data:

(1) A category $\mathcal{G}(U)$ given for all open $U \subset \mathcal{S}$, the restriction functors $r_{U V}: \mathcal{G}(U) \rightarrow \mathcal{G}(V)$ given for any morphism $V \rightarrow U$, and natural isomorphisms of functors $s_{U V W}: r_{V W} \circ$ $r_{U V} \Rightarrow r_{U W}$ given for each $W \rightarrow V \rightarrow U$ and satisfying the transitivity conditions.

(2) The structure of $\mathcal{F}(U)$-torsor on each $\operatorname{Hom}_{\mathcal{G}(U)}(x, y)$ compatible with the $r_{U V}$ and such that the composition of morphisms is bi-additive.

These data have to satisfy the local uniqueness and gluing properties for which we refer to [6].

By a sheaf of $\mathcal{F}$-groupoids we will mean a sheaf of categories $\mathcal{C}$ on $\mathcal{S}$ (so both $\mathrm{Ob} \mathcal{C}$ and Mor $\mathcal{C}$ are sheaves of sets) in which each $\operatorname{Hom}_{\mathcal{C}(U)}(x, y)$ is either empty or is made into an $\mathcal{F}(U)$-torsor

$4^{\mathrm{e}}$ SÉRIE - TOME $40-2007-\mathrm{N}^{\circ} 1$ 
so that the composition is $\mathcal{F}$-bilinear. A sheaf $\mathcal{C}$ of $\mathcal{F}$-groupoids is called locally connected if locally on $S$ all the $\operatorname{ObC}(U)$ and $\operatorname{Hom}_{\mathcal{C}(U)}(x, y)$ are nonempty.

Each sheaf of $\mathcal{F}$-groupoids can be seen as a fibered category over $\mathcal{S}$, in fact it is a pre-stack, see, e.g., [23, Definition 3.1]. Recall that for any pre-stack $\mathcal{C}$ there is a canonical associated stack $\mathcal{C}^{\sim}$. See, e.g., loc. cit. Lemma 3.2. If $\mathcal{C}$ is a locally connected sheaf of $\mathcal{F}$-groupoids, then $\mathcal{C}^{\sim}$ is an $\mathcal{F}$-gerbe. We will refer to this procedure as "gerbification" (analog of sheafification).

As well known (see, e.g., [6]), the set formed by $\mathcal{F}$-gerbes up to equivalence is identified with $H^{2}(\mathcal{S}, \mathcal{F})$. Given an $\mathcal{F}$-gerbe $\mathcal{G}$, we denote by $[\mathcal{F}] \in H^{2}(S, \mathcal{F})$ its class. Given a sheaf $\mathcal{C}$ of $\mathcal{F}$-groupoids, we denote by $[\mathcal{C}]$ the class of the corresponding gerbe.

\section{Ind-schemes and their cohomology}

Let $F$ be a field. By $\mathbf{S c h}_{F}$ we denote the category of $F$-schemes. If $S$ is a scheme, then by $S_{\text {Zar }}$ we denote the underlying topological space with the Zariski topology.

As in [20], by an ind-scheme over $F$ we will mean an ind-object of $\mathbf{S c h}_{F}$ of the form " $\lim " Y^{\alpha}$ where $\alpha$ runs in a filtered poset $A, Y^{\alpha}$ are quasicompact schemes (not necessarily of finite type) and the structural maps $Y^{\alpha} \rightarrow Y^{\alpha^{\prime}}$ are closed embeddings. We denote by $\mathbf{I s c h}_{F}$ the category of ind-schemes over $F$. In the case $F=\mathbb{C}$ we write simply Sch and Isch.

As with any ind-object, we can identify an ind-scheme $Y=$ "lim" $Y^{\alpha}$ with the functor $h_{Y}=\lim \operatorname{Hom}\left(-, Y^{\alpha}\right)$ on $\mathbf{S c h}_{F}$. Let $\mathbf{A f f}_{F} \subset \mathbf{S} \mathbf{S h}_{F}$ be the subcategory of affine schemes, dual to $\mathbf{A l g}_{F}$, the category of commutative $F$-algebras. It is well known that $Y$ is determined by the restriction of $h_{Y}$ to $\mathbf{A f f}_{F}$ which can be regarded as a covariant functor on $\mathbf{A} \lg _{F}$.

The subcategory of ind-affine ind-schemes over $F$ is dual to that of pro-algebras over $F$, i.e., pro-objects " $\lim ^{-} A_{\alpha}$ over $\mathbf{A l g}_{F}$ with the structure morphisms being surjective. Such a pro-algebra can be identified with the complete topological algebra $\lim A_{\alpha}$ with the projective limit topology (compare [18]). For a pro-algebra $A=$ “lim” $A_{\alpha}$ we denote by $\operatorname{Spf}(A)=$ "lim”Spec $\left(A_{\alpha}\right)$ the corresponding ind-affine ind-scheme. We also define the Laurent series algebra of $A$ as follows:

$$
A((t))=\lim _{\longleftarrow} A_{\alpha}((t)) .
$$

Note that elements of $A((t))$ can be represented by formal series $\sum_{i=-\infty}^{\infty} a_{i} t^{i}$ with $a_{i}$ being elements of the topological algebra $\lim A_{\alpha}$. These series are allowed to be infinite in both directions with the condition that the $\overleftarrow{a_{i} \rightarrow 0}$ as $i \rightarrow-\infty$.

For an ind-scheme $Y=$ " $\lim$ " $Y^{\alpha}$ we denote by $Y_{\mathrm{Zar}}=\lim Y_{\mathrm{Zar}}^{\alpha}$ the inductive limit of the $Y_{\mathrm{Zar}}^{\alpha}$ in the category of topological spaces. Thus an open set in $\overrightarrow{\mathrm{Zar}}_{\mathrm{Zar}}$ is the same as a compatible system of Zariski open subsets $U^{\alpha} \subset Y^{\alpha}$.

2.1. Definition. - A natural sheaf on the category of $\mathbf{S c h}_{F}$ is a system $\mathcal{F}$ consisting of a Zariski sheaf $\mathcal{F}_{Y}$ given for each scheme $Y$ and a morphism of sheaves $u_{f}: f^{-1}\left(\mathcal{F}_{Y^{\prime}}\right) \rightarrow \mathcal{F}_{Y}$ given for each morphism of schemes $f: Y \rightarrow Y^{\prime}$ and satisfying the condition $u_{f \circ g}=u_{f} \circ u_{g}$ for any composable pair of morphisms $f, g$.

2.2. Examples. - The sheaves $\mathcal{O}_{Y}$ form a natural sheaf. If $\Phi$ is any covariant functor from rings to abelian groups, then the sheaves $\Phi\left(\mathcal{O}_{U}\right)$ (obtained by sheafifying the presheaves $U \mapsto \Phi(\mathcal{O}(U)))$ form a natural sheaf. In particular, the sheaves $\Omega_{Y}^{1}$ (Kähler differentials) form natural sheaves. 
2.3. Proposition. - Let $Z$ be an ind-scheme.

(a) Any natural sheaf $\mathcal{F}$ gives rise to a sheaf of pro-abelian groups on $Z_{Z \text { ar }}$ denoted by $\mathcal{F}_{Z}$. In particular, $\mathcal{O}_{Z}$ is a sheaf of pro-algebras (or complete topological algebras) on $Z_{\mathrm{Zar}}$.

(b) Let $Z$ be represented as " $\lim$ " $Z^{\alpha}$. Then we have a natural map

$$
H^{i}\left(Z_{\mathrm{Zar}}, \mathcal{F}_{X}\right) \rightarrow \lim _{\longleftarrow} H^{i}\left(Z^{\alpha}, \mathcal{F}_{Z^{\alpha}}\right)
$$

which is an isomorphism for $i=0$.

Proof. - The Grothendieck site $Z_{\mathrm{Zar}}^{\alpha}$ is the category of open sets in $Z^{\alpha}$ with morphisms being embeddings. So whenever $\alpha \leqslant \alpha^{\prime}$ we have a functor $Z_{\mathrm{Zar}}^{\alpha^{\prime}} \rightarrow Z_{\mathrm{Zar}}^{\alpha}$ given by intersecting open sets with $Z^{\alpha}$. Then, as a category, we have $Z_{\text {Zar }}=\lim Z_{\text {Zar }}^{\alpha}$. Accordingly, the Čech complex calculating $H^{\bullet}\left(Z, \mathcal{F}_{X}\right)$ is the projective limit of Čech complexes calculating $H^{\bullet}\left(Z^{\alpha}, \mathcal{F}_{Z^{\alpha}}\right)$.

Note that a morphism $f: Y \rightarrow Z$ of ind-schemes gives a morphism of locally ringed spaces $\left(Y_{\mathrm{Zar}}, \mathcal{O}_{Y}\right) \rightarrow\left(Z_{\mathrm{Zar}}, \mathcal{O}_{Z}\right)$ and therefore we have an inverse image map on sheaf cohomology as above. Further, a result of Haboush [18] identifies $\mathbf{I s c h}_{F}$ with a full subcategory in the category of topologically locally ringed spaces. This subcategory consists of objects of the form $\left(Y_{\mathrm{Zar}}, \mathcal{O}_{Y}\right)$. Equivalently, one can say that this subcategory consists of objects locally isomorphic to "affine topological schemes", i.e., topologically ringed spaces of the form $\left(\operatorname{Spf}(A)_{\mathrm{Zar}}, \mathcal{O}_{\operatorname{Spf}(A)}\right)$ for a pro-algebra $A$. We will use both points of view.

2.4. Definition. - Let a natural sheaf $\mathcal{F}$ on the category of schemes be given and $Y=$ " $\lim$ " $Y^{\alpha}$ be an ind-scheme. An $\mathcal{F}$-gerbe on $Y$ is a system of $\mathcal{F}^{\alpha}$-gerbes $\mathcal{G}^{\alpha}$ on $Y^{\alpha}$ together with equivalences of gerbes

$$
\phi_{\alpha \alpha^{\prime}}: \mathcal{G}^{\alpha} \rightarrow i_{\alpha \alpha^{\prime}}^{*}\left(\mathcal{G}^{\alpha^{\prime}}\right)
$$

and isomorphisms of functors

$$
\psi_{\alpha_{1} \alpha_{2} \alpha_{3}}: \phi_{\alpha_{1} \alpha_{3}} \Rightarrow \phi_{\alpha_{1} \alpha_{2}} \circ \phi_{\alpha_{2} \alpha_{3}}, \quad \alpha_{1} \leqslant \alpha_{2} \leqslant \alpha_{3},
$$

satisfying the coherence conditions for any $\alpha_{1} \leqslant \alpha_{2} \leqslant \alpha_{3} \leqslant \alpha_{4}$.

Thus an $\mathcal{F}$-gerbe on $Y$ has a class in $H^{2}\left(Y_{\text {Zar }}, \mathcal{F}\right)$ similar to the case of usual schemes.

\section{The determinantal gerbe of a locally free $\mathcal{O}_{S}((t))$-module}

Let $S$ be a scheme and $\mathcal{E}$ be a locally free $\mathcal{O}_{S}((t))$-module of rank $N$. We call a lattice in $\mathcal{E}$ a sheaf of $\mathcal{O}_{S}[[t]]$-submodules $\mathcal{F} \subset \mathcal{E}$ such that, Zariski locally on $S$, the pair $(\mathcal{F}, \mathcal{E})$ is isomorphic to $\left(\mathcal{O}_{S}[[t]]^{N}, \mathcal{O}_{S}((t))^{N}\right)$.

3.1. Proposition. - Assume that $S$ is quasicompact. Let $\mathcal{F}_{1}, \mathcal{F}_{2}$ are two lattices.

(a) Zariski locally on $S$, there exist $a, b \in \mathbb{Z}$ such that

$$
t^{a} \mathcal{F}_{1} \subset \mathcal{F}_{2} \subset t^{b} \mathcal{F}_{1}
$$

(b) If $\mathcal{F}_{1} \subset \mathcal{F}_{2}$ then $\mathcal{F}_{2} / \mathcal{F}_{1}$ is locally free over $\mathcal{O}_{S}$ of finite rank.

Proof. - (a) By quasicompactness of $S$ it is enough to prove the statement locally, in particular, to assume that $\mathcal{E}=\mathcal{O}_{S}((t))^{N}$, that $\mathcal{F}_{2}=\mathcal{O}_{S}[[t]]^{N}$ and that $\mathcal{F}_{1}=A \cdot \mathcal{F}_{2}$ where

$4^{e}$ SÉRIE - TOME $40-2007-\mathrm{N}^{\circ} 1$ 
$A \in G L_{N}(\mathcal{O}(S)((t)))$. Our statement follows by taking $a$ to be the negative of the maximal order of poles of the coefficients of $A$ and $b$ to be the maximal order of poles of the coefficients of $A^{-1}$.

(b) Again, it is enough to work locally and assume that $S=\operatorname{Spec}(R)$ is affine. Then the existence of $A$ implies that for any affine $U \subset S$ the $\mathcal{O}(U)[[t]]$-submodule $\mathcal{F}_{i}(U) \subset \mathcal{E}(U)$ is coprojective over $\mathcal{O}(U)$, i.e. $\mathcal{E}(U) / \mathcal{F}_{i}(U)$ is projective. It follows that the sequence of $\mathcal{O}_{S}$-modules

$$
0 \rightarrow \mathcal{F}_{2} / \mathcal{F}_{1} \rightarrow \mathcal{E} / \mathcal{F}_{1} \rightarrow \mathcal{E} / \mathcal{F}_{2} \rightarrow 0
$$

splits over $\mathcal{O}_{S}$, so $\mathcal{F}_{2} / \mathcal{F}_{1}$ is locally a direct summand of a projective $\mathcal{O}_{S}$-module, hence it is itself projective. The finiteness of the rank follows from (a).

\subsection{The twisted affine Grassmannian}

Consider the contravariant functor $\gamma: \mathbf{S c h}_{S} \rightarrow$ Sets taking $p: T \rightarrow S$ to the set of $\mathcal{O}_{T}[[t]]$-lattices $\mathcal{F} \subset p^{*} \mathcal{E}$. Here

$$
p^{*} \mathcal{E}=p^{-1} \mathcal{E} \otimes_{p^{-1} \mathcal{O}_{S}((t))} \mathcal{O}_{T}((t))
$$

is the inverse image in the sense of morphisms of ringed spaces

$$
\left(T, \mathcal{O}_{T}((t))\right) \rightarrow\left(S, \mathcal{O}_{S}((t))\right)
$$

3.2.1. PROPOSITION. - The functor $\gamma$ is represented by an ind-proper formally smooth indscheme over $S$, denoted by $\mathcal{G} r(S, \mathcal{E})$.

Proof. - It is enough to prove the statement Zariski locally on $S$. In particular, we can assume that $S=\operatorname{Spec}(R)$ is affine and that $\mathcal{E}=\mathcal{O}_{S}((t))^{N}$ is free. For any $a<b \in \mathbb{Z}$ let $\gamma_{a, b}$ be the sub-functor of $\gamma$ taking $T$ to the set of lattices

$$
\mathcal{F} \subset p^{*} \mathcal{E}=\mathcal{O}_{T}((t))^{N}
$$

that are contained between $t^{a} \mathcal{O}_{T}[[t]]^{N}$ and $t^{b} \mathcal{O}_{T}[[t]]^{b}$. Let also $\xi_{a, b}$ be the functor taking $T$ to the set of $\mathcal{O}_{S}$-submodules contained between $t^{a} \mathcal{O}_{T}[[t]]^{N}$ and $t^{b} \mathcal{O}_{T}[[t]]^{b}$. Then, clearly, $\xi_{a, b}$ is represented by the relative Grassmannian of subspaces (of all dimensions) in the vector bundle $t^{b} \mathcal{O}_{S}[[t]]^{N} / t^{a} \mathcal{O}_{S}[[t]]^{N}$ and $\gamma_{a, b}$ is defined inside this relative Grassmannian by closed conditions of being an $\mathcal{O}_{S}[[t]]$-submodule. So $\gamma_{a, b}$ is represented by a proper scheme $\mathcal{G} r_{a, b}(S, \mathcal{E})$ of finite type over $S$. Finally, as $\gamma=\lim _{a, b} \gamma_{a, b}$ in the category of functors, we conclude that it is representable by the ind-scheme $\mathcal{G} r(S, \mathcal{E})=$ " $\lim ^{\longrightarrow}{ }_{a, b} \mathcal{G} r_{a, b}(S, \mathcal{E})$.

\subsection{The $\mathcal{O}^{\times}$-groupoid structure}

Proposition 3.1 can be reformulated as follows.

3.3.1. LEMMA. - Given an open set $U \subset S$ and two $U$-points $\mathcal{F}_{1}, \mathcal{F}_{2}$ of $\mathcal{G} r(S, \mathcal{E})$, we can find Zariski locally on $U$ a third point $\mathcal{F}^{\prime}$ such that as a submodule $\mathcal{F}^{\prime}$ is contained in each $\mathcal{F}_{i}$ and the quotients $\mathcal{F}_{i} / \mathcal{F}^{\prime}$ are free over $\mathcal{O}_{U}$ of finite rank.

We define the relative determinantal line bundle as usual, i.e.,

$$
\left(\mathcal{F}_{1} \mid \mathcal{F}_{2}\right)=\bigwedge^{\max }\left(\mathcal{F}_{1} / \mathcal{F}^{\prime}\right) \otimes \bigwedge^{\max }\left(\mathcal{F}_{2} / \mathcal{F}^{\prime}\right)^{-1}
$$


See $[1,27]$ for instance. This is a line bundle that is independent (up to a unique isomorphism) of the choice of $\mathcal{F}^{\prime}$ and thus it is well defined by gluing for any two $U$-points $\mathcal{F}_{1}, \mathcal{F}_{2}$. It is also clear that for three $U$-points we have a canonical isomorphism

$$
\left(\mathcal{F}_{1} \mid \mathcal{F}_{2}\right) \otimes\left(\mathcal{F}_{2} \mid \mathcal{F}_{3}\right) \rightarrow\left(\mathcal{F}_{1} \mid \mathcal{F}_{3}\right) .
$$

3.3.2. LEMMA. - The $\mathcal{O}_{U}^{\times}$-torsors corresponding to the line bundles $\left(\mathcal{F}_{1} \mid \mathcal{F}_{2}\right)$ make $\mathcal{G} r(S, \mathcal{E})$ into the sheaf of objects of a sheaf of $\mathcal{O}^{\times}$-groupoids on $S$. This sheaf of groupoids is locally connected and hence gives rise to an $\mathcal{O}_{S}^{\times}$-gerbe.

We denote this gerbe by $\operatorname{Det}(\mathcal{E})$. Note that $[\mathcal{D} e t(\mathcal{E})] \in H^{2}\left(S, \mathcal{O}_{S}^{\times}\right)$.

\section{Loop ind-schemes and the evaluation maps}

\subsection{The restriction of scalars}

From now on, all rings will be assumed to contain the field $\mathbb{C}$ of complex numbers and by (ind-)schemes we will mean (ind-)schemes over $\mathbb{C}$.

We define a functor

$$
\mathcal{R}=\mathcal{R}_{\mathbb{C}}^{\mathbb{C}((t))}: \operatorname{Aff}_{\mathbb{C}((t))} \rightarrow \text { Isch, }
$$

called the restriction of scalars (from $\mathbb{C}((t))$ to $\mathbb{C}$ ). Given an affine scheme $Y$ over $\mathbb{C}((t))$, the ind-scheme $\mathcal{R} Y$ represents the following functor on $\mathrm{Alg}$ :

$$
\operatorname{Hom}_{\mathbf{I s c h}}(\operatorname{Spec} A, \mathcal{R} Y)=\operatorname{Hom}_{\mathbf{S c h}_{\mathbb{C}(t))}}(\operatorname{Spec} A((t)), \mathcal{R} Y) .
$$

It is well known that this functor is indeed represented by an ind-affine ind-scheme. Compare [11, Section 6.3.4], where $\mathcal{R} Y$ is denoted by $\mathcal{L} Y$.

4.1.2. Proposition. - (a) The functor $\mathcal{R}$ commutes with finite projective limits, in particular, with fiber products.

(b) The functor $\mathcal{R}$ also commutes with disjoint unions.

Proof. - (a) This follows directly from (4.1.1) as, for any $S$,

$$
\operatorname{Hom}\left(S, \lim _{\longleftarrow} Z_{i}\right)=\lim \operatorname{Hom}\left(S, Z_{i}\right) .
$$

(b) Obvious.

\subsection{The evaluation map}

Let $Y \in \mathbf{A f f}_{\mathbb{C}((t))}$. We define a morphism of ringed Grothendieck sites

$$
\epsilon:\left((\mathcal{R} Y)_{\mathrm{Zar}}, \mathcal{O}_{\mathcal{R} Y}((t))\right) \rightarrow\left(Y_{\mathrm{Zar}}, \mathcal{O}_{Y}\right)
$$

called the evaluation map. The underlying morphism of sites

$$
\varepsilon_{\sharp}:(\mathcal{R} Y)_{\mathrm{Zar}} \rightarrow Y_{\mathrm{Zar}}
$$

is better defined by considering them as Grothendieck sites and putting, for a Zariski open $U \subset Y$

$$
\epsilon^{-1}(U)=\mathcal{R} U \subset \mathcal{R} Y .
$$

$4^{e}$ SÉRIE - TOME $40-2007-N^{\circ} 1$ 
4.2.1. Proposition. - (a) $\mathcal{R} U$ is Zariski open in $\mathcal{R} Y$.

(b) The correspondence $U \mapsto \mathcal{R} U$ commutes with disjoint unions and intersections.

(c) If $U=\bigcup_{\alpha} U_{\alpha}$ is a covering in $Y_{\mathrm{Zar}}$, then $\left\{\mathcal{R} U_{\alpha}\right\}$ form a covering of $\mathcal{R} U$ in $(\mathcal{R} Y)_{\mathrm{Zar}}$.

Proof. - (a) Let $Y=\operatorname{Spec} R, R \in \mathbf{A l g}_{\mathbb{C}((t))}$. A basis of open sets in $Y_{\text {Zar }}$ is formed by the "principal affine sets"

$$
U_{f}=\{x \in Y: f(x) \neq 0\}, \quad f \in R .
$$

It is enough to prove that $\mathcal{R} U_{f}$ is open in $\mathcal{R} Y$. Let $y$ be a $\mathbb{C}$-point of $\mathcal{R} Y$ and $y(t)$ be the corresponding $\mathbb{C}((t))$-point of $Y$. Then $y \in \mathcal{R} U_{f}$ if and only if $y(t) \in U_{f}$, i.e. $f(y(t)) \neq 0$ as an element of $\mathbb{C}((t))$. Writing $f(y(t))=\sum_{n \in \mathbb{Z}} a_{n} t^{n}$, we realize each $a_{n}$ as a global section of $\mathcal{O}_{\mathcal{R} Y}$. So

$$
U_{a_{n}}=\left\{y \in \mathcal{R} Y: a_{n}(y) \neq 0\right\}
$$

is Zariski open in $\mathcal{R} Y$ and

$$
\mathcal{R} U_{f}=\bigcup_{n \in \mathbb{Z}} U_{a_{n}}
$$

is open as well.

(b) Follows from Proposition 4.1.2.

(c) If $U=\bigcup_{\alpha} U_{\alpha}$, then for any field $F$ we have $U(F)=\bigcup_{\alpha} U_{\alpha}(F)$. Taking $F=\mathbb{C}((t))$, we get

$$
(\mathcal{R} U)(\mathbb{C})=U(\mathbb{C}((t)))=\bigcup_{\alpha} U_{\alpha}(\mathbb{C}((t)))=\bigcup_{\alpha}\left(\mathcal{R} U_{\alpha}\right)(\mathbb{C})
$$

Let $S=\operatorname{Spec} A$ be an affine scheme over $\mathbb{C}$, and $f: S \rightarrow \mathcal{R} Y$ be a morphism. Let $\tilde{f}: \operatorname{Spec} A((t)) \rightarrow Y$ be the morphism of $\mathbb{C}((t))$-schemes corresponding to $f$ by (4.1.1). It induces a ring homomorphism

$$
\tilde{f}^{*}: \Gamma\left(Y, \mathcal{O}_{Y}\right) \rightarrow A((t))=\Gamma\left(S, \mathcal{O}_{S}((t))\right) .
$$

By performing the same construction for affine open sets $U \subset Y$ (forming a basis of topology of $\left.Y_{\mathrm{Zar}}\right)$ we get a morphism of sheaves of rings on $S$

$$
\epsilon_{S, f}^{b}: f^{-1} \epsilon_{\sharp}^{-1} \mathcal{O}_{Y} \rightarrow \mathcal{O}_{S}((t)) .
$$

These morphisms, taken for all $S, f$ as above, give rise to a morphism of sheaves of rings on $\mathcal{R} Y$

$$
\epsilon^{b}: \epsilon_{\sharp}^{-1} \mathcal{O}_{Y} \rightarrow \mathcal{O}_{\mathcal{R} Y}((t)) .
$$

This finishes the definition of $\epsilon$.

\subsection{The transgression map}

We now discuss an algebraic construction which is an analog of $\int_{\Sigma / B}$ in (0.3.3).

Let $A$ be a commutative ring. We denote by $K_{j}(A)$ the $j$-th Quillen K-group, so $K_{*}(A)$ is a graded commutative ring. Let also $K_{j}^{M}(A)$ be the $j$-th Milnor K-group. Recall [12] that $K_{*}^{M}(A)$ is defined as the graded commutative ring generated by symbols $l(a), a \in A^{\times}$in degree 1 which are subject to the relations: 


$$
\begin{aligned}
& l(a b)=l(a)+l(b), \quad a, b \in A^{\times} ; \\
& l(a) l(1-a)=0, \quad a, 1-a \in A^{\times} ; \\
& l(a) l(-a)=0, \quad a \in A^{\times} .
\end{aligned}
$$

In particular, $K_{0}^{M}(A)=\mathbf{Z}$ and $K_{1}^{M}(A)=A^{\times}$. One denotes the element $l\left(a_{1}\right) \ldots l\left(a_{n}\right) \in$ $K_{n}^{M}(A)$ by $\left\{a_{1}, \ldots, a_{n}\right\}$. There is a canonical ring homomorphism

$$
\lambda_{*}: K_{*}^{M}(A) \rightarrow K_{*}(A),
$$

taking $a \in A^{\times}$to its class in $K_{1}(A)$. The following fact follows from the results of [29].

4.3.2. THEOREM. - If $A$ is a local ring with an infinite residue field, then $\lambda_{i}: K_{i}^{M}(A) \rightarrow$ $K_{i}(A)$ is an isomorphism for $i \leqslant 2$.

Let $S$ be a scheme over C. By sheafifying the presheaf $U \mapsto K_{j}^{M}(\mathcal{O}(U))$ we get a sheaf $K_{j}^{M}\left(\mathcal{O}_{S}\right)$. Since the stalks of $\mathcal{O}_{S}$ are local $\mathbf{C}$-algebras, the above theorem implies:

4.3.3. Corollary. - The sheaves $K_{j}^{M}\left(\mathcal{O}_{S}\right)$ and $K_{j}\left(\mathcal{O}_{S}\right)$ are identified for $j \leqslant 2$.

We now recall the definition of the homomorphism

$$
\partial: K_{2}^{M} A((t)) \rightarrow A^{\times}=K_{1}^{M} A,
$$

called the Contou-Carrère symbol [8]. To do this, it suffices to define $\partial\{a(t), b(t)\} \in A^{*}$ for any two units $a(t), b(t) \in A((t))^{\times}$and show that it satisfies the relations for $K_{2}^{M}$ above. We recall the following lemma, see [8] (1.3) and [10] (2.9).

4.3.4. Lemma. - (a) Assume that $\operatorname{Spec}(A)$ is connected, i.e., $A$ is not a product of rings. Then any invertible $a(t)=\sum_{i \gg-\infty}^{\infty} a_{i} t^{i} \in A((t))^{\times}$satisfies the following property: There exist $n=\operatorname{ord}(a) \in \mathbb{Z}$ such that $a_{n}$ is invertible and $a_{i}, i<n$ are nilpotent.

(b) The correspondence $a \mapsto \operatorname{ord}(a)$ is a homomorphism $A((t))^{\times} \rightarrow \mathbb{Z}$.

(c) If $A$ is arbitrary and $a(t) \in A((t))^{\times}$, then there is a decomposition of $A$ into a finite product of rings such that the description from (a) applies to each factor.

This can be reformulated as a statement describing the ind-scheme $G L_{1}((t))=\mathcal{R}\left(G L_{1} /\right.$ $\mathbb{C}((t)))$. By definition, an $A$-point of $G L_{1}((t))$ is an element of $G L_{1}(A((t)))=A((t))^{\times}$.

4.3.5. COROLlary. - We have an identification of ind-schemes:

$$
G L_{1}((t))=\mathbb{Z} \times \mathbb{C}^{\times} \times \operatorname{Spec} \mathbb{C}\left[a_{1}, a_{2}, \ldots\right] \times \operatorname{Spf} \mathbb{C}\left[\left[a_{-1}, a_{-2}, \ldots\right]\right] .
$$

Here $\operatorname{Spf} \mathbb{C}\left[\left[a_{-1}, a_{-2}, \ldots\right]\right]$ is, by definition, the ind-scheme

$$
\text { “ } \underline{\lim }{ }_{\epsilon} \operatorname{Spec} \mathbb{C}\left[a_{-1}, a_{-2}, \ldots\right] /\left(a_{i}^{\epsilon_{i}+1}=0, i<0\right),
$$

where $\epsilon$ runs over sequences $\epsilon=\left(\epsilon_{-1}, \epsilon_{-2}, \ldots\right), \epsilon_{i} \in \mathbb{Z}_{+}$, almost all $\epsilon_{i}=0$.

The above identification corresponds to writing an element of $A((t))$ in Lemma 4.3.4(a) as a product

$$
a(t)=t^{n} \cdot a_{0} \cdot\left(1+\sum_{i=1}^{\infty} a_{i} t^{i}\right) \cdot\left(1+\sum_{i=-\infty}^{-1} a_{i} t^{i}\right)
$$

$4^{e}$ SÉRIE - TOME $40-2007-N^{\circ} 1$ 
with $a_{0} \in A^{\times}, a_{i}, i>0$, arbitrary and $a_{i}, i<0$, nilpotent.

Let us now describe the Contou-Carrère symbol.

In virtue of Lemma 4.3.4(c) we will assume in the remainder of this section that $A$ is not a product. Let $a(t), b(t) \in A((t))^{\times}$be two units with $\operatorname{ord}(a(t))=n, \operatorname{ord}(b(t))=m$. The ContouCarrère symbol of $a(t)$ and $b(t)$ is defined by (see [10] (2.9)):

$$
\partial\{a(t), b(t)\}:=(-1)^{m n} b_{m}^{-n} \exp \left(\operatorname{Res}\left(\frac{d a}{a} \cdot \log \left(\frac{b(t)}{b_{m} t^{m}}\right)\right)\right) .
$$

Here $b(t) / b_{m} t^{m}$ is the sum of 1 and a topologically nilpotent element so its logarithm is a well defined element of $A((t))$.

4.3.7. Remark. - For any commutative ring $A$ one can obtain the map $\partial_{i}^{Q}: K_{i} A((t)) \rightarrow$ $K_{i-1} A$ on Quillen K-functors using the localization theorem for singular varieties ([28], Ch. 9). It seems that the image of $\partial_{2}^{Q}$ on elements $\lambda_{2}\{a(t), b(t)\} \in K_{2} A((t))$ for $a(t), b(t) \in A((t))^{\times}$is in fact equal to the Contou-Carrère symbol although we could not find a reference for this in the literature.

Let now $Y$ be a scheme over $\mathbb{C}((t))$. For any sheaf $\mathcal{E}$ of $\mathcal{O}_{Y}$-modules we have a sheaf

$$
\mathcal{R E}=\epsilon^{*} \mathcal{E}=\epsilon_{\sharp}^{-1} \mathcal{E} \otimes_{\epsilon_{\sharp}^{-1} \mathcal{O}_{Y}} \mathcal{O}_{\mathcal{R} Y}((t))
$$

of $\mathcal{O}_{\mathcal{R} Y}((t))$-modules. In particular, $\mathcal{R O}_{Y}=\mathcal{O}_{\mathcal{R} Y}((t))$.

The morphism of ringed spaces $\epsilon$ induces homomorphisms of abelian groups

$$
\epsilon^{*}: H^{i}\left(Y, K_{j}^{M}\left(\mathcal{O}_{Y}\right)\right) \rightarrow H^{i}\left(\mathcal{R} Y, K_{j}^{M}\left(\mathcal{O}_{\mathcal{R} Y}((t))\right)\right) .
$$

Sheafifying the Contou-Carrère symbol on $\mathcal{R} Y$, we get a morphism of sheaves of abelian groups

$$
\partial: K_{2}^{M}\left(\mathcal{O}_{\mathcal{R} Y}((t))\right) \rightarrow K_{1}^{M}\left(\mathcal{O}_{\mathcal{R} Y}\right)=\mathcal{O}_{\mathcal{R} Y}^{\times}
$$

Composing it with $\epsilon^{*}$ we get, for any $i \geqslant 0$, a homomorphism

$$
\tau=\tau_{i}: H^{i}\left(Y, K_{2}\left(\mathcal{O}_{Y}\right)\right) \rightarrow H^{i}\left(\mathcal{R} Y, \mathcal{O}_{\mathcal{R} Y}^{\times}\right)
$$

which we call the transgression.

\section{Chern classes and the local Riemann-Roch theorem}

\subsection{Reminder on simplicial geometry}

Recall that a simplicial object in a category $\mathbf{C}$ is a family $X_{\bullet}=\left(X_{n}\right)_{n \geqslant 0}$ of objects of $\mathbf{C}$ together with face and degeneracy morphisms

$$
d_{i}: X_{n} \rightarrow X_{n-1}, \quad s_{i}: X_{n} \rightarrow X_{n+1}, \quad i=0,1, \ldots, n
$$

satisfying the standard identities. 
5.1.1. Examples. - (a) Let $F$ be a field and $\mathbf{C}=\mathbf{S c h}_{F}$. Let $S$ be a scheme and $U=\left\{U_{\alpha}\right\}_{\alpha}$ be a Zariski open covering. The nerve $\mathcal{N}_{\bullet} U$ is a simplicial scheme with

$$
\mathcal{N}_{n} U=\coprod_{\alpha_{0}, \alpha_{1}, \ldots, a_{n} \in A}\left(U_{\alpha_{0}} \cap \cdots \cap U_{\alpha_{n}}\right) .
$$

(b) Let $\mathbf{C}=\mathbf{I s c h}_{F}$ and $G$ be a group ind-scheme over $F$. The classifying space of $G$ is the simplicial ind-scheme $B_{\bullet} G$ with $B_{n} G=G^{n}$.

Let $X_{\bullet}$ be a simplicial topological space. A sheaf of abelian groups $\mathcal{F}_{\bullet}$ on $X_{\bullet}$ is a family of sheaves $\mathcal{F}_{n}$ on $X_{n}$ together with morphisms

$$
d_{i}^{-1} \mathcal{F}_{n-1} \rightarrow \mathcal{F}_{n}, \quad s_{i}^{-1} \mathcal{F}_{n+1} \rightarrow \mathcal{F}_{n}
$$

compatible with the identities among $d_{i}, s_{i}$. The groups

$$
\mathbb{H}^{i}\left(X_{\bullet}, \mathcal{F}_{\bullet}\right)
$$

are defined as the cohomology groups of the double complex formed by the Čech complexes of $\mathcal{F}_{n}$ on $X_{n}$ for $n \geqslant 0$, see [14], so that one has a spectral sequence

$$
E_{2}=H^{i}\left(X_{j}, \mathcal{F}_{j}\right) \quad \Rightarrow \quad \mathbb{H}^{i-j}\left(X_{\bullet}, \mathcal{F}_{\bullet}\right)
$$

5.1.2. Examples. - (a) If $X_{\bullet}$ is a simplicial ind-scheme, then we have the simplicial topological space $\left(X_{\bullet}\right)_{\mathrm{Zar}}$, and $\mathcal{O}=\mathcal{O}_{X_{\bullet}}=\left(\mathcal{O}_{X_{n}}\right)$ is a sheaf of rings on $\left(X_{\bullet}\right)_{\mathrm{Zar}}$. Therefore $K_{j}(\mathcal{O})=\left(K_{j}\left(\mathcal{O}_{X_{n}}\right)\right)$ is a sheaf of abelian groups on $\left(X_{\bullet}\right)_{\mathrm{Zar}}$. Similarly we have the sheaf on $\left(X_{\bullet}\right)$ Zar

$$
K_{j}(\mathcal{O}((t)))=\left(K_{j}\left(\mathcal{O}_{X_{n}}((t))\right)\right) .
$$

(b) If $S$ is a scheme, $U=\left\{U_{\alpha}\right\}_{\alpha}$ is a Zariski open cover, and $\mathcal{F}$ is a sheaf on $S_{\text {Zar }}$, we get a simplicial sheaf $\mathcal{F}_{\bullet}$ on $\mathcal{N}_{\bullet} U$ with

$$
\left.\mathcal{F}_{n}\right|_{U_{\alpha_{0}} \cap \cdots \cap U_{\alpha_{n}}}=\left.\mathcal{F}\right|_{U_{\alpha_{0}} \cap \cdots \cap U_{\alpha_{n}}} .
$$

The Mayer-Vietoris property for sheaf cohomology implies that

$$
\mathbb{H}^{i}\left(\mathcal{N}_{\bullet} U, \mathcal{F}_{\bullet}\right)=H^{i}(S, \mathcal{F})
$$

\subsection{The Chern classes of Goncharov}

Let $F$ be a field and $G L_{N} / F$ be the algebraic group $G L_{N}$ considered as a scheme over $F$. Then $B \bullet\left(G L_{N} / F\right)$ is a simplicial $F$-scheme. Goncharov [14] has constructed classes

$$
c_{i} \in \mathbb{H}^{i}\left(B_{\bullet}\left(G L_{N} / F\right), K_{i}^{M}(\mathcal{O})\right)
$$

which we will call the universal Chern classes. They lift the Chern classes of Gillet [13] to Milnor K-theory. In this paper we will be working only with $c_{i}$ for $i=1,2$ when, by Corollary 4.3.3, there is no difference between $K_{i}$ and $K_{i}^{M}$ for sheaves of local $\mathbb{C}$-algebras.

5.2.1. Examples. - Let $S$ be a smooth scheme over $F$ and $\mathcal{E}$ a locally free sheaf of $\mathcal{O}_{S^{-}}$ modules of rank $N$. Choosing a Zariski open covering $U=\left\{U_{\alpha}\right\}_{\alpha}$ of $S$ such that $\mathcal{E}$ is free

$4^{\text {e }}$ SÉRIE - TOME $40-2007-\mathrm{N}^{\circ} 1$ 
over each $U_{\alpha}$, and a system of trivializations

$$
\varphi=\left(\varphi_{\alpha}:\left.\mathcal{E}\right|_{U_{\alpha}} \rightarrow \mathcal{O}_{U_{\alpha}}^{N}\right)
$$

one gets the system of transition functions

$$
\varphi_{\alpha, \beta}: U_{\alpha} \cap U_{\beta} \rightarrow G L_{N}
$$

These functions give a morphism of simplicial schemes

$$
\tilde{\varphi}: \mathcal{N}_{\bullet} U \rightarrow B_{\bullet}\left(G L_{N} / F\right) .
$$

The inverse image

$$
\widetilde{\varphi}^{*} c_{i} \in \mathbb{H}^{i}\left(\mathcal{N}_{\bullet} U, K_{i}^{M}(\mathcal{O})\right)=H^{i}\left(S, K_{i}^{M}\left(\mathcal{O}_{S}\right)\right)
$$

is nothing but $c_{i}(E)$, the $K$-theoretic Chern class of $\mathcal{E}$. To be precise, for a Noetherian regular scheme $S$ we have $H^{i}\left(S, K_{i}\left(\mathcal{O}_{S}\right)\right)=C H^{i}(S)$ which is the Chow group of cycles of codimension $i$, see [28]. The image of $\widetilde{\varphi}^{*} c_{i}$ under the map $\lambda_{i}$ of (4.3.1) is the $i$-th Chern class with values in the Chow group.

Let us now take $F=\mathbb{C}((t))$. The group scheme $G L_{N} / \mathbb{C}((t))$ gives the group ind-scheme

$$
G L_{N}((t)):=\mathcal{R}\left(G L_{N} / \mathbb{C}((t))\right)
$$

over $\mathbb{C}$. The evaluation map from (4.2) gives a morphism of ringed simplicial topological spaces

$$
\epsilon:\left(B \bullet G L_{N}((t)), \mathcal{O}((t))\right) \rightarrow\left(B \bullet\left(G L_{N} / \mathbb{C}((t))\right), \mathcal{O}\right) .
$$

Therefore we have the classes

$$
c_{i}^{((t))}=\epsilon^{*} c_{i} \in \mathbb{H}^{i}\left(B_{\bullet}\left(G L_{N}((t))\right), K_{i}^{M}(\mathcal{O}((t)))\right) .
$$

Let now $S$ be a scheme over $\mathbb{C}$ and $\mathcal{E}$ be a locally free sheaf of $\mathcal{O}_{S}((t))$-modules of rank $N$. Trivializing $\mathcal{E}$ over open sets from a covering $U$, as in Example 5.2.1, we get a morphism of simplicial ind-schemes over $\mathbb{C}$

$$
\tilde{\varphi}: \mathcal{N}_{\bullet} U \rightarrow B \bullet G L_{N}((t))
$$

which induces the classes

$$
c_{i}^{((t))}(\mathcal{E}):=\tilde{\varphi}^{*} c_{i}^{((t))} \in H^{i}\left(S, K_{i}^{M}\left(\mathcal{O}_{S}((t))\right)\right) .
$$

\subsection{The local Riemann-Roch theorem}

Let $S$ be a scheme, $\mathcal{E}$ a locally free sheaf of $\mathcal{O}_{S}((t))$-modules of rank $N$. Then we have the second Chern character class

$$
c h_{2}^{((t))}(\mathcal{E})=\frac{1}{2}\left(c_{1}^{((t))}(\mathcal{E})\right)^{2}-c_{2}^{((t))}(\mathcal{E}) \in H^{2}\left(S, K_{2}^{M} \mathcal{O}_{S}((t))\right) \otimes \mathbb{Z}\left[\frac{1}{2}\right] .
$$

On the other hand we have the determinantal class

$$
[\mathcal{D} e t(\mathcal{E})] \in H^{2}\left(S, O_{S}^{\times}\right)
$$


The Contou-Carrère symbol gives a homomorphism of sheaves

$$
\partial: K_{2}\left(\mathcal{O}_{S}((t))\right) \rightarrow \mathcal{O}_{S}^{\times} .
$$

It yields a map

$$
\partial: H^{2}\left(S, K_{2}\left(\mathcal{O}_{S}((t))\right)\right) \rightarrow H^{2}\left(S, \mathcal{O}_{S}^{\times}\right)
$$

5.3.1. ThEOREM. - We have $[\operatorname{Det}(\mathcal{E})]=\partial\left(\operatorname{ch}_{2}^{((t))}(\mathcal{E})\right)$ in $H^{2}\left(S, \mathcal{O}_{S}^{\times}\right) \otimes \mathbb{Z}\left[\frac{1}{2}\right]$.

To prove the theorem, we realize $[\operatorname{Det}(\mathcal{E})]$ as

$$
[\mathcal{D} e t(\mathcal{E})]=\tilde{\varphi}^{*}[\mathcal{D} e t]
$$

for a universal class

$$
[\mathcal{D} e t] \in \mathbb{H}^{2}\left(B_{\bullet} G L_{N}((t)), \mathcal{O}^{\times}\right) .
$$

Here $\tilde{\varphi}$ is as in (5.2.3). Then the theorem reduces to the statement about the universal classes:

$$
[\mathcal{D} e t]=\partial\left(\frac{1}{2}\left(c_{1}^{((t))}\right)^{2}-c_{2}^{((t))}\right) \in \mathbb{H}^{2}\left(B \bullet G L_{N}((t)), \mathcal{O}^{\times}\right) \otimes \mathbb{Z}\left[\frac{1}{2}\right]
$$

We start with a standard lemma.

5.3.5. LEMMA. - Let $G$ be a group ind-scheme over $\mathbb{C}$. Then $\mathbb{H}^{2}\left(B, G, \mathcal{O}^{\times}\right)$is identified with the group of central extensions

$$
1 \rightarrow \mathbb{C}^{\times} \rightarrow \widetilde{G} \rightarrow G \rightarrow 1
$$

(in the category of group ind-schemes).

Recall now the definition of the determinantal central extension

$$
1 \rightarrow \mathbb{C}^{\times} \rightarrow \widetilde{G L_{N}}((t)) \rightarrow G L_{N}((t)) \rightarrow 1 .
$$

The group ind-scheme $\widetilde{G L_{N}}((t))$ represents the following functor on Alg:

$$
\begin{aligned}
& \operatorname{Hom}\left(\operatorname{Spec}(A), \widetilde{G L} L_{N}((t))\right) \\
& \quad=\left\{(g, u): g \in G L_{N} A((t)), u \in \operatorname{det}\left(g A[[t]]^{N}: A[[t]]^{N}\right) \text { invertible }\right\} .
\end{aligned}
$$

Here "invertible" means "an element of a projective $A$-module of rank one which does not vanish over any prime ideal". The group law is defined as in [1, Section 5]. Explicitly, we set

$$
(g, u) \cdot\left(g^{\prime}, u^{\prime}\right)=\left(g g^{\prime}, u \cdot\left(g \cdot u^{\prime}\right)\right),
$$

where $g \cdot u^{\prime}$ is the element of $\operatorname{det}\left(g g^{\prime} A[[t]]^{N}: g A[[t]]^{N}\right)$ obtained from $u^{\prime}$ via the action of $G L_{N} A((t))$ on $A((t))^{N}$. Note that $u \cdot\left(g \cdot u^{\prime}\right)$ belongs to

$$
\operatorname{det}\left(g A[[t]]^{N}: A[[t]]^{N}\right) \otimes \operatorname{det}\left(g g^{\prime} A[[t]]^{N}: g A[[t]]^{N}\right),
$$

which is canonically identified with

$$
\operatorname{det}\left(g g^{\prime} A[[t]]^{N}: A[[t]]^{N}\right) .
$$

$4^{e}$ SÉRIE - TOME $40-2007-\mathrm{N}^{\circ} 1$ 
We now define $[\mathcal{D} e t]$ in (5.3.3) to be the class of the extension (5.3.6). It is clear from the definition of $[\mathcal{D} e t(\mathcal{E})]$ via the determinantal gerbe that the equality (5.3.2) holds. We concentrate therefore on the proof of (5.3.4), which, by Lemma 5.3.5, is a statement comparing two central extensions of $G L_{N}((t))$.

\subsection{Proof of Theorem 5.3.1}

Let $\widetilde{G}$ be the central extension of $G L_{N}((t))$ corresponding to the class $2[\operatorname{Det}(\mathcal{E})]-$ $\partial\left(2 c h_{2}^{((t))}\right)$. We will prove that $\widetilde{G}$ is trivial.

5.4.1. PROPOSITION. - We have that $\widetilde{G}$ is trivial on the sub-ind-group scheme $T((t))$ where $T \subset G L_{N}$ is the subgroup of diagonal matrices.

Proof. - To emphasize the dependence of our classes on $N$, let us denote them by $[\mathcal{D} e t]_{N}$ and $2 c h_{2, N}^{((t))}$. Notice that both these classes are additive with respect to the block diagonal embeddings

$$
\varphi_{N_{1}, N_{2}}: G L_{N_{1}}((t)) \times G L_{N_{2}}((t)) \rightarrow G L_{N_{1}+N_{2}}((t))
$$

i.e.

$$
\varphi_{N_{1}, N_{2}}^{*}\left(\left[\mathcal{D} e t_{N_{1}+N_{2}}\right]\right)=\left[\mathcal{D} e t_{N_{1}}\right]+\left[\mathcal{D} t_{N_{2}}\right]
$$

and similarly for $c h_{2}^{((t))}$. Indeed, for [Det] it follows right away from (5.3.6) and for $2 c h_{2}^{((t))}$ by the standard additivity property of the Chern character. Therefore it is enough to establish the lemma for $N=1$. Then $2 c h_{2}^{((t))}=\left(c_{1}^{((t))}\right)^{2}$. Let us first identify $c_{1}^{((t))}$. Notice that the hypercohomology spectral sequence gives a homomorphism

$$
\xi: \operatorname{Hom}\left(G L_{1}((t)), G L_{1}((t))\right) \rightarrow \mathbb{H}^{1}\left(B \bullet G L_{1}((t)), \mathcal{O}((t))^{\times}\right)
$$

where Hom on the left is the set of homomorphisms of group ind-schemes. The next lemma is clear.

5.4.2. LEMMA. - We have that $c_{1}^{((t))}=\xi(\mathrm{Id})$.

Lemma 5.4.2 implies that $\partial\left(c_{1}^{((t))}\right)^{2}$ is the image of the tame symbol map $G L_{1}((t)) \times$ $G L_{1}((t)) \rightarrow \mathbb{C}^{\times}$under the homomorphism

$$
Z^{2}\left(G L_{1}((t)), \mathbb{C}^{\times}\right) \rightarrow \mathbb{H}^{2}\left(B_{\bullet} G L_{1}((t)), \mathcal{O}^{\times}\right)
$$

where $Z^{2}$ is the set of 2-cocycles that are morphisms of ind-schemes. To finish the proof of Proposition 5.4.1, it suffices to establish the following.

5.4.3. LEMMA. - In the group $\mathbb{H}^{2}\left(B . G L_{1}((t)), \mathcal{O}^{\times}\right) \otimes \mathbb{Z}\left[\frac{1}{2}\right]$ we have $2[$ Det $]=\partial\left(\left(c_{1}^{((t))}\right)^{2}\right)$.

Proof. - We start with a general remark. Let $E, A$ be abelian groups and $\widetilde{E}$ be a central extension

$$
1 \rightarrow A \rightarrow \widetilde{E} \rightarrow E \rightarrow 1
$$

As any central extension of any group (abelian or not), it corresponds to a class in $H^{2}(E, A)$. The well-known procedure to represent this class by a cocycle is as follows. One takes for each $x \in E$ a lifting $\widetilde{x} \in \widetilde{E}$ and writes the cocycle

$$
\gamma(x, y)=\widetilde{x} \widetilde{y} \cdot(\widetilde{x y})^{-1} .
$$


On the other hand, since $E$ is abelian, one can form the commutator pairing

$$
c(x, y)=\widetilde{x} \widetilde{y} \widetilde{x}^{-1} \widetilde{y}^{-1},
$$

which, as any bilinear pairing on any abelian group, can also be considered as a 2-cocycle. It is clear that

$$
c(x, y)=\gamma(x, y) \gamma(y, x)^{-1} .
$$

On the other hand, $\gamma(x, y) \gamma(y, x)$ is the coboundary of the 1-cochain $x \mapsto \gamma(x, x)$. This means that the class if $c(x, y)$ is equal to twice the class of $\gamma(x, y)$.

We now apply the above to the case where $E$ is the group scheme $G L_{1}((t)), A=\mathbb{C}^{\times}$ and $\widetilde{E}$ is the central extension corresponding to the determinantal gerbe. To be precise, we take a commutative algebra $A$ and apply the above to groups of $A$-points. It follows from the results of [2] that for $a(t), b(t) \in A((t))^{\times}$the commutator pairing $c(a(t), b(t))$ is equal to $(-1)^{\text {ord }}(a) \operatorname{ord}(b)$ times the Contou-Carrère symbol of $a(t), b(t)$. To be precise, in [2] the authors treated the case of an artinian local ring $A$. However, both the commutator pairing and the Contou-Carrère symbol are regular functions, i.e., morphisms of ind-schemes

$$
G L_{1}((t)) \times G L_{1}((t)) \rightarrow \mathbb{C} .
$$

It follows from the explicit description of $G L_{1}((t))$ as an ind-scheme (Corollary 4.3.5) that two such morphisms are equal if and only if they are equal on the set of points with values in any artinian local ring.

It follows that after inverting 2, the cohomology class describing the extension is one half of the class of the Contou-Carrère symbol, i.e., $2[\mathcal{D} e t]=\partial\left(\left(c_{1}^{((t))}\right)^{2}\right)$ as claimed in Lemma 5.4.3. This also finishes the proof of Proposition 5.4.1.

5.4.4. LEMMA. - The extension $\widetilde{G}$ is trivial on the subgroup scheme $G L_{N}[[t]]$.

Proof. - For $[\mathcal{D} e t]$ the triviality on $G L_{N}[[t]]$ follows from (5.3.6) and for $\partial\left(2 c h_{2}^{((t))}\right)$ from the fact that $\partial$ is trivial on $K_{2}(A[[t]])$ for any ring $A$.

5.4.5. LeMmA. - The extension $\widetilde{G}$ is trivial on the subgroup ind-scheme $S L_{N}((t))$.

Proof. - Let $\mathcal{G}=S L_{N}((t)) / S L_{N}[[t]]$ be the affine Grassmannian for $S L_{N}$. Because of Lemma 5.4.4 the extension $\left.\tilde{G}\right|_{S L_{N}((t))}$ (viewed as a multiplicative $\mathcal{O}^{\times}$-torsor on $S L_{N}((t))$ ) descends to an $\mathcal{O}^{\times}$-torsor on $\mathcal{G}$, i.e. to a line bundle which we denote by $\mathcal{L}$. Recall that $\mathcal{P} i(\mathcal{G})=\mathbb{Z}$. See [22, Proposition 13.2.19] for instance. Let $T_{S L} \subset T$ be the intersection $T \cap S L_{N}$. By Lemma 5.4.1 the line bundle $\mathcal{L}$ is equivariant with respect to $T_{S L}((t))$. However, it is known that any nontrivial line bundle on $\mathcal{G}$ is equivariant with respect to a nontrivial central extension of $S L_{N}((t))$, and this extension remains nontrivial on $T_{S L}((t))$. See op. cit. and the references there for details. So $T_{S L}((t))$-equivariance implies that $\mathcal{L}$ is trivial. This implies that the projection

$$
\left.\widetilde{G}\right|_{S L_{N}((t))} \rightarrow S L_{N}((t))
$$

splits as a morphism of ind-schemes and so $\left.\widetilde{G}\right|_{S L_{N}((t))}$ is given by a 2-cocycle

$$
\eta: S L_{N}((t)) \times S L_{N}((t)) \rightarrow \mathbb{C}^{\times}
$$

which is a morphism of ind-schemes. The fact that $\eta$ is trivial follows from the next lemma.

$4^{e}$ SÉRIE - TOME $40-2007-\mathrm{N}^{\circ} 1$ 
5.4.6. LEMMA. - We have that $\Gamma\left(S L_{N}((t)), \mathcal{O}^{\times}\right)=\mathbb{C}^{\times}$.

Proof. - As well-known, any unimodular matrix over any field, in particular, over the field $\mathbb{C}((t))$ can be factored as a product of elementary matrices $e_{i j}(a), a \in \mathbb{C}((t)), i, j=1,2, \ldots, N$, $i \neq j$. This means that there is an integer $M$ and a sequence of pairs of indices $\left(i_{\nu}, j_{\nu}\right)$, $\nu=1,2, \ldots, M$, such that every matrix as above can be written as

$$
e_{i_{1}, j_{1}}\left(a_{1}\right) e_{i_{2}, j_{2}}\left(a_{2}\right), \ldots, e_{i_{M}, j_{M}}\left(a_{M}\right), \quad a_{\nu} \in \mathbb{C}((t)) .
$$

Let now $\mathbb{C}((t))=\mathcal{R}\left(\mathbb{A}^{1} / \mathbb{C}((t))\right)$ be the ind-scheme whose set of $\mathbb{C}$-points is $\mathbb{C}((t))$. As it is an inductive limit of affine spaces over $\mathbb{C}$, we have

$$
\Gamma\left(\underline{\mathbb{C}((t))}, \mathcal{O}^{\times}\right)=\mathbb{C}^{\times} .
$$

On the other hand, the construction above gives a surjective morphism of ind-schemes

$$
\underline{\mathbb{C}((t))^{M}} \rightarrow S L_{N}((t))
$$

A nonvanishing function $f$ on $S L_{N}((t))$ gives then a nonvanishing function $\tilde{f}$ on $\mathbb{C}((t))^{M}$ which must be constant. So $f$ is constant as well. Lemmas 5.4.6 and 5.4.5 are proved.

Now we prove the triviality of $\widetilde{G}$ on the whole $G L_{N}((t))$. We represent $G L_{N}((t))$ as a semidirect product

$$
G L_{N}((t))=G L_{1}((t)) \ltimes S L_{N}((t))
$$

where $G L_{1}((t))$ consists of matrices $\operatorname{diag}(a, 1, \ldots, 1)$. As for any semidirect product (see [7, Section 1.7]), the category of central extensions of $G L_{N}((t))$ becomes identified with the category of triples $\left(E, E^{\prime}, \rho\right)$ where:

- $E$ is a central extension of $S L_{N}((t))$,

- $E^{\prime}$ is a central extension of $G L_{1}((t))$,

- $\rho$ is an action of $G L_{1}((t))$ on $E$ lifting the action on $S L_{N}((t))$ by conjugation.

Consider the triple $\left(E, E^{\prime}, \rho\right)$ corresponding to our extension $\widetilde{G}$. By the above lemmas, both $E$ and $E^{\prime}$ are trivial. So $\rho$ is a morphism of group ind-schemes

$$
\rho: G L_{1}((t)) \times S L_{N}((t)) \rightarrow \mathbb{C}^{\times} .
$$

By Lemma 5.4.5 the map $\rho$ does not depend on the second variable. So it is uniquely determined by its restriction to $G L_{1}((t)) \times T_{S L}((t))$. This restriction, however, classifies the extension $\left.\widetilde{G}\right|_{T((t))}$ which is trivial by Lemma 5.4.1. So $\rho$ is trivial and so is $\widetilde{G}$. Theorem 5.3.1 is proved.

\section{Application to the anomaly of the loop space and to chiral differential operators}

\subsection{Reminder on the formal loop space}

Let $X$ be an affine scheme over $\mathbb{C}$. The ind-scheme $\mathcal{R}(X \otimes \mathbb{C}((t)))$ will be denoted by $\widetilde{\mathcal{L}} X$. Thus

$$
\operatorname{Hom}_{\mathbf{I s c h}}(\operatorname{Spec}(A), \widetilde{\mathcal{L}} X)=\operatorname{Hom}_{\mathbf{S c h}}(\operatorname{Spec}(A((t))), X) .
$$


Let also $\mathcal{L}^{0} X \subset \widetilde{\mathcal{L}} X$ be the subscheme such that

$$
\operatorname{Hom}_{\mathbf{S c h}}\left(\operatorname{Spec}(A), \mathcal{L}^{0} X\right)=\operatorname{Hom}_{\mathbf{S c h}}(\operatorname{Spec}(A[[t]]), X) .
$$

The formal completion of $\widetilde{\mathcal{L}} X$ along $\mathcal{L}^{0} X$ will be denoted by $\mathcal{L} X$ and called the formal loop space of $X$.

For a commutative ring $A$ let $A((t)) \sqrt{ } \subset A((t))$ be the subring of series $\sum_{n \in \mathbb{Z}} a_{n} t^{n}$ such that for any $n<0$ the element $a_{n} \in A$ is nilpotent. Thus

$$
\operatorname{Hom}_{\mathbf{I s c h}}(\operatorname{Spec}(A), \mathcal{L} X)=\operatorname{Hom}_{\operatorname{Sch}}\left(\operatorname{Spec}\left(A((t))^{\vee}\right), X\right) .
$$

It is well known that the scheme $\mathcal{L}^{0} X$ can be defined for any variety $X$, affine or not, and the condition (6.1.2) holds. See [5] for details. Further, for any scheme $S$ we have

$$
\operatorname{Hom}_{\mathbf{S c h}}\left(S, \mathcal{L}^{0} X\right)=\operatorname{Hom}_{\mathbf{L r s}}\left(\left(S, \mathcal{O}_{S}[[t]]\right),\left(X, \mathcal{O}_{X}\right)\right),
$$

where Lrs is the category of locally ringed spaces.

It was shown in [20] that $\mathcal{L} X$ can also be defined for an arbitrary $X$ and, in addition to (6.1.3), we have, for any scheme $S$,

$$
\operatorname{Hom}_{\mathbf{I s c h}}(S, \mathcal{L} X)=\operatorname{Hom}_{\mathbf{L r s}}\left(\left(S, \mathcal{O}_{S}((t)) \sqrt{ }\right),\left(X, \mathcal{O}_{X}\right)\right)
$$

Thus, for any $f: S \rightarrow \mathcal{L} X$, we will denote by

$$
f_{\sharp}: S \rightarrow X, \quad f^{b}: f_{\sharp}^{-1} \mathcal{O}_{X} \rightarrow \mathcal{O}_{S}((t))^{\sqrt{ }}
$$

the corresponding morphism of ringed spaces. Thus we have a diagram

$$
X \stackrel{p}{\longleftarrow} \mathcal{L}^{0} X \stackrel{i}{\hookrightarrow} \mathcal{L} X
$$

The ind-scheme $\mathcal{L} X$ being a formal neighborhood of $\mathcal{L} X$, it is completely determined by the sheaf of topological rings $\mathcal{O}_{\mathcal{L} X}$ on $\left(\mathcal{L}^{0} X\right)_{\mathrm{Zar}}=(\mathcal{L} X)_{\mathrm{Zar}}$.

\subsection{The evaluation map}

Let $X$ be an arbitrary scheme over $\mathbb{C}$, and $U \subset X$ be an affine open subset. We have then the evaluation map

$$
\epsilon_{U}:\left((\widetilde{\mathcal{L}} U)_{\mathrm{Zar}}, \mathcal{O}_{\tilde{\mathcal{L}} U}((t))\right) \rightarrow\left((U \otimes \mathbb{C}((t)))_{\mathrm{Zar}}, \mathcal{O}_{U \otimes \mathbb{C}((t))}\right)
$$

from Section 5. Consider the composition of $\epsilon_{U}$ with the embedding

$$
\left((\mathcal{L} U)_{\mathrm{Zar}}, \mathcal{O}_{\mathcal{L} U}((t))\right) \subset\left((\tilde{\mathcal{L}} U)_{\mathrm{Zar}}, \mathcal{O}_{\tilde{\mathcal{L}} U}((t))\right)
$$

and the projection (morphism of schemes)

$$
U \otimes \mathbb{C}((t)) \rightarrow U
$$

The resulting morphism of ringed spaces will be denoted by

$$
e v_{U}:\left(\mathcal{L}^{0} U, \mathcal{O}_{\mathcal{L} U}((t))\right) \rightarrow\left(U, \mathcal{O}_{U}\right)
$$

$4^{\mathrm{e}}$ SÉRIE - TOME $40-2007-\mathrm{N}^{\circ} 1$ 
Since for $U^{\prime} \subset U$ we have $e v_{U^{\prime}}=\left.e v_{U}\right|_{\mathcal{L}^{0} U^{\prime}}$, we have that the $e v_{U}, U \subset X$, glue together into a morphism of ringed spaces

$$
e v=e v_{X}:\left(\mathcal{L}^{0} X, \mathcal{O}_{\mathcal{L} X}((t))\right) \rightarrow\left(X, \mathcal{O}_{X}\right)
$$

which we also call the evaluation map. Its underlying morphism of topological spaces is $p: \mathcal{L}^{0} X \rightarrow X$. It is the algebro-geometric analog of the evaluation map

$$
S^{1} \times \operatorname{Map}\left(S^{1}, X\right) \rightarrow X
$$

for the space of smooth loops.

\subsection{The bundles $\mathcal{E}_{\mathcal{L}}$}

Let $X$ be a $\mathbb{C}$-scheme and $\mathcal{E}$ a quasi-coherent sheaf of $\mathcal{O}_{X}$-modules. We denote

$$
\mathcal{E}_{\mathcal{L}}=e v^{*}(\mathcal{E})=p^{-1} \mathcal{E} \otimes_{p^{-1} \mathcal{O}_{X}} \mathcal{O}_{\mathcal{L} X}((t)) .
$$

This is a $\mathcal{O}_{\mathcal{L} X}((t))$-module. The relation between $e v$ and $\epsilon_{U}, U \subset X$ affine open, allows us to describe $\mathcal{E}_{\mathcal{L}}$ via $\epsilon_{U}$. Namely, let $\mathcal{E} \otimes \mathbb{C}((t))$ be the quasicoherent sheaf on $X \otimes \mathbb{C}((t))$ obtained by extension of scalars. Then

$$
\mathcal{E}_{\mathcal{L}}=\left.\epsilon_{U}^{*}(\mathcal{E} \otimes \mathbb{C}((t)))\right|_{\mathcal{L} X}
$$

For an ind-scheme $Z$ one defines the sheaf $\Omega_{Z}^{1}$ on $Z_{Z \text { Zar }}$ as in [11]. Then, Lemma 6.5 of op. cit. together with (6.3.2) imply the following fact.

6.3.3. TheOREM. - Let $X$ be a smooth $\mathbb{C}$-variety. Then $\Omega_{\mathcal{L} X}^{1}=\Omega_{X, \mathcal{L}}^{1}$.

\subsection{The RR theorem for the bundles $\mathcal{E}_{\mathcal{L}}$}

By definition, for every scheme $S$ and any morphism $f: S \rightarrow \mathcal{L} X$ the preimage of $\mathcal{E}_{\mathcal{L}}$ under $f$ is equal to

$$
\mathcal{E}_{\mathcal{L}, f}=f_{\sharp}^{-1}(\mathcal{E}) \otimes_{f_{\sharp}^{-1}\left(\mathcal{O}_{X}\right)} \mathcal{O}_{S}((t)) .
$$

Thus, the determinantal classes of the $\mathcal{E}_{\mathcal{L}, f}$ give rise to a class

$$
\left[\operatorname{Det}\left(\mathcal{E}_{\mathcal{L}}\right)\right] \in H^{2}\left(\mathcal{L} X, \mathcal{O}_{\mathcal{L} X}^{\times}\right)
$$

6.4.1. THEOREM. - We have that $\left[\operatorname{Det}\left(\mathcal{E}_{\mathcal{L}}\right)\right]=\partial\left(e v^{*}\left(\operatorname{ch}_{2}(\mathcal{E})\right)\right)$ in $H^{2}\left(\mathcal{L} X, \mathcal{O}_{\mathcal{L} X}^{\times}\right) \otimes \mathbb{Z}\left[\frac{1}{2}\right]$, where

$$
e v^{*}: H^{2}\left(X, K_{2}\left(\mathcal{O}_{X}\right)\right) \rightarrow H^{2}\left(\mathcal{L} X, K_{2}\left(\mathcal{O}_{\mathcal{L} X}((t))\right)\right)
$$

is the morphism induced by ev on $K_{2}$ and

$$
\partial: H^{2}\left(\mathcal{L} X, K_{2}\left(\mathcal{O}_{\mathcal{L} X}((t))\right)\right) \rightarrow H^{2}\left(\mathcal{L} X, \mathcal{O}_{\mathcal{L} X}^{\times}\right)
$$

is the boundary map.

Proof. - Follows from the local Riemann-Roch theorem 5.3.1 applied to any $(S, f)$. 


\subsection{Consequences for the gerbe of chiral differential operators}

Let $X$ be a smooth $\mathbb{C}$-variety. The category $\mathbf{D}_{\mathcal{L} X}$ of right $\mathcal{D}$-modules on $\mathcal{L} X$ was constructed in [20] as an abstract category, but not as a category of sheaves. In the forthcoming paper [21] it is shown that a trivialization $\tau$ of the gerbe $\operatorname{Det}\left(\Omega_{\mathcal{L} X}^{1}\right)$ gives a functor

$$
\gamma_{\tau}: \mathbf{D}_{\mathcal{L} X} \rightarrow \mathbf{S h}_{X}
$$

In particular, applying $\gamma_{\tau}$ to the object

$$
i_{!} p^{!}\left(\omega_{X}\right) \in \mathbf{D}_{\mathcal{L} X}
$$

one gets a sheaf of chiral differential operators. Thus, Theorem 6.4 .1 provides a conceptual explanation of the result of $[16,17]$ relating the class of the gerbe of CDO with $c h_{2}\left(\Omega_{X}^{1}\right)$.

\section{REFERENCES}

[1] Arbarello E., De Concini C., Kac V., The infinite wedge representation and the reciprocity law for algebraic curves, Part 1, in: Proc. Sympos. Pure Math., vol. 49, Amer. Math. Soc., Providence, RI, 1989, pp. 171-190.

[2] Anderson G., PAblos Romo F., Simple proofs of classical explicit reciprocity laws on curves using determinantal groupoids over an artinian local ring, Comm. Algebra 32 (2004) 79-102.

[3] Bloch S., $K_{2}$ and algebraic cycles, Ann. of Math. 99 (1974) 349-379.

[4] Bressler P., Kapranov M., Tsygan B., Vasserot É., Riemann-Roch for real varieties, in preparation.

[5] Bosch S., LÜtkebohmert W., Raynaud M., Néron Models, Springer-Verlag, Berlin/New York, 1990

[6] BREEN L., On the classification of 2-gerbes and 2-stacks, Astérisque 225 (1994).

[7] Brylinski J.-L., Deligne P., Central extensions of reductive groups by $K_{2}$, Publ. Math. IHÉS 94 (2001) 5-85.

[8] CONTOU-CARRÈre C., Jacobienne locale, groupe de bivecteurs de Witt universel et symbole modéré, C. R. Acad. Sci. Paris, Série I 318 (1994) 743-746.

[9] Deligne P., Le déterminant de la cohomologie, Contemp. Math. 67 (1987) 93-177.

[10] Deligne P., Le symbole modéré, Publ. Math. IHÉS 73 (1991) 147-181.

[11] DRINFELD V., Infinite-dimensional vector bundles in algebraic geometry (an introduction), math.AG/0309155.

[12] Elbaz-Vincent Ph., Mueller-Stach S., Milnor K-theory of rings, higher Chow groups and applications, Invent. Math. 148 (2002) 177-206.

[13] Gillet H., Riemann-Roch theorems for higher algebraic K-theory, Adv. Math. 40 (1981) 203-289.

[14] Goncharov A.B., Explicit construction of characteristic classes, in: Adv. Soviet Math., vol. 16, Amer. Math. Soc., Providence, RI, 1993, pp. 169-210.

[15] Denef J., LOESER F., Germs of arcs on singular algebraic varieties and motivic integration, Invent. Math. 135 (1999) 201-232.

[16] Gorbounov V., Malikov F., Schechtman V., Gerbes of chiral differential operators, Math. Res. Lett. 7 (2000) 55-66.

[17] Gorbounov V., Malikov F., Schechtman V., Gerbes of chiral differential operators. II. Vertex algebroids, Invent. Math. 155 (2004) 605-680.

[18] HabOUSH W., Infinite-dimensional algebraic geometry: algebraic structures on $p$-adic groups and their homogeneous spaces, Tohoku Math. J. 57 (2005) 65-117.

[19] KAC V., Vertex Algebras for Beginners, University Lecture Series, vol. 10, Amer. Math. Soc., Providence, RI, 1996.

$4^{e}$ SÉRIE - TOME $40-2007-\mathrm{N}^{\circ} 1$ 
[20] Kapranov M., VAsserot É., Vertex algebras and the formal loop space, Publ. Math. IHÉS 100 (2004) 209-269.

[21] KAPRANOV M., VASSEROT É., Formal loops III: Chiral differential operators, in preparation.

[22] Kumar S., Kac-Moody Groups, Their Flag Varieties and Representation Theory, Progress in Math., vol. 204, Birkhäuser, Basel, 2002.

[23] Laumon G., Moret-Bailly L., Champs algébriques, A Series of Modern Surveys in Mathematics, vol. 39, Springer-Verlag, Berlin/New York, 2000.

[24] Matsumoto H., Sur les sous-groupes arithmétiques des groupes semi-simples déployés, Ann. Sci. École Norm. Sup. 2 (1969) 1-62.

[25] Moore C.C., Group extensions of $p$-adic and adelic linear groups, Publ. Math. IHÉS 35 (1968) 157222.

[26] Malikov F., Schechtman V., Vaintrob A., Chiral de Rham complex, Comm. Math. Phys. 204 (1999) 439-473.

[27] Pressley A., Segal G., Loop Groups, Oxford Univ. Press, London, 1986.

[28] SRInivas V., Algebraic K-theory, Birkhäuser, Basel, 1996.

[29] VAN DER KALlen W., The $K_{2}$ of rings with many units, Ann. Sci. École Norm. Sup. 10 (1977) 473-515.

Mikhail KAPRANOV

Department of Mathematics, Yale University,

10 Hillhouse Avenue,

New Haven, CT 06520, USA

E-mail: mikhail.kapranov@yale.edu

Éric VASSEROT

Université Paris 7

Département de Mathématiques,

175 rue du Chevaleret,

75013 Paris, France

E-mail: vasserot@math.jussieu.fr 